\title{
RECENT OPERATIONAL EXPERIENCE WITH THE FERMILAB TEVATRON
}

John L. Crawford and Robert C. Mau

Fermi National Accelerator Laboratory*

$$
\text { P. 0. Box } 500
$$

Batavia, Illinois 60510

\section{Abstract}

This report summarizes the performance of the Fermilab accelerator complex, particularly the Tevatron, during the two years since the 1987 Particle Accclerator Conference. The accelerator has provided $800 \mathrm{GeV}$ protons for one fixed-target run and $900 \mathrm{GeV}$ protons and anti-protons for two collider runs during this period. Major accomplishments, as well as some of the operational problem areas, are highlighted.

\section{Introduction}

During the last two years, for the first time since 1982, the accelerator complex has returned to its primary mission of providing beam for High Energy Physies experiments; multi-month shutdowns have become a thing of the past, with 82 of the past 104 weeks being devoted to HEP. The Tevatron has matured to become a reliable link in the acceleration process, exhibiting little of the cantankerous behavior which some predicted six years ago.

The accelerator now alternates between fixed target mode at $800 \mathrm{GeV}$, and collider mode at $1.8 \mathrm{TeV}$ center-of-mass; each run lasts on the order of $8-9$ months, with a one month turn-around in between.

\section{Fixed-Target Operation}

Accelerator start up for the 1987 fixed-target run began on June 1; the run ended on February 15, 1988 with some 2870 hours of HEP accumulated. Operation was at $800 \mathrm{GeV}$ with a 23 second flattop during which slow spill was delivered to some 17 separate experiments. There were also three fast pulses $(2-3$ milliseconds and $\sim 1.5 \times 1012$ each) equally spaced during flattop. The average machine intensity was raised to greater than $1 \times 1013$ protons per pulse within a few weeks and eventually leveled of $f$ at about $1.6 \times 1013$; the peak intensity extracted from the Tevatron was $1.8 \times 1013$ with $2.19 \times 1018$ protons accelerated during the 35 week run.

Near the end of the run, the Switchyard Beam Position Monitor (BPM) system and associated application software was used as a position servo to maintain beam position through the Meson beamline. Once this system is made fully operational, it promises much improved position stability throughout the Switchyard.

\section{Reliability}

0verall machine reliability was $\sim 59 \%$; of the roughly 2000 hours of downtime, fully one-half was due to Tevatron magnel replacements; there were seven separate instances of magnet failures, each of which cost roughly one week of running time (more on this subject later).

The next largest Tevatron downtime contributor was recovery time from quenches of the superconducting magnets - some 210 hours; this was mainly due to the three high intensity fast spills for several Neutrino area experiments. The large instantaneous beam loss in the fast spill process produced a large number of flattop quenches in the region of the extraction channe1 at A11; about two months into the run, fear of component damage led to tightening of loss abort tolerances in the A11 area. Thereafter, many fast spills were aborted, rather than being allowed to continue to the point of quenching the magnets. Although the number of aborts dramatically increased, overall reliability improved as an abort cost one cycle of beam (about one minute), whereas a high field quench required approximately one hour of recovery time.

\section{Tevatron Fixed Target Operation} integrated Intensity at $\mathrm{BOO} \mathrm{GeV}$

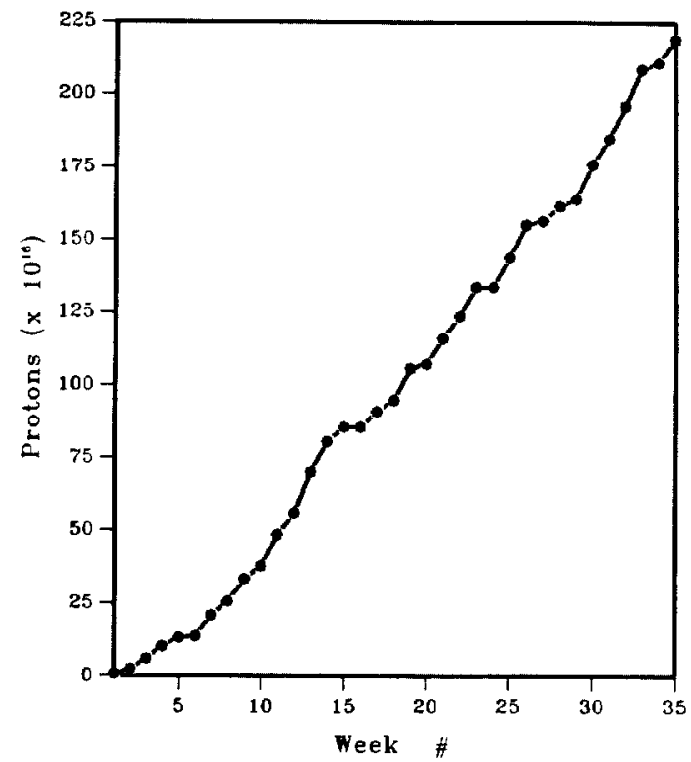

Figure 1: Integrated intensity from $6 / 1 / 87-2 / 15 / 88$.

Figure 1 gives a weekly breakdown of accelerator performance; the magnet failures show up clearly in weeks $5,15,17,20,24,27$, and 34 . In addition to the magnet failures, an elusive ground fault appeared in the Al1 region at about week 10; fault hunting and component substitution occupied many hours until the offending magnet was replaced during the failure of week 24. Examination of weekly reliability figures indicates that, had it not been for the unfortunate spate of magnet failures, accelerator reliability for the run would have been on the order of $70 \%$.

\section{Cryogenics}

The operation of the Central Helium Liquefier and the satellite refrigerators around the ring deserve special mention. Prior to commissioning the Tevatron, it was assumed that the cryogenic system would be by far the largest contributor to machine downtime, however the $1985 \mathrm{fixed-target}$ run showed that cryogenic 
downtime was comparable to that of other major subsystems. This trend continued, and in fact improved, during 1987 and 1988, with all cryogenic systems (including CHL) being responsible for less than $4 \%$ of the total downtime.

\section{No Scheduled Maintenance}

The number of equipment failures during this run was nearly $1 / 3$ less than during the 1985 run; this is particularly interesting in light of an experiment that was tried for the first time during the 1987 run - the elimination of scheduled preventive maintenance periods. In previous runs the machine had been shut off for approximately two days every other week, under the assumption that certain failures could be predicted and prevented by regular scheduled maintenance. For this run it was decided to keep the accelerator operating until a serious enough failure occurred to allow for widespread system maintenance. Although the aforementioned magnet failures gave us more unscheduled downtime than we would have liked, the experiment qualitatively was a success, and it was decided to continue the policy in future runs.

\section{Tevalron Magnet Repair}

Following the fixed-target run, the accelerator was shut down for three months of maintenance and development work. The primary mission during this period was to inspect and repair as many Tevatron dipoles as possible; inspection consisted of visually examining the magnet lead areas using a borescope and utilizing two industrial gamma-ray sources to take $x-$ ray pictures of the magnet ends.1 The magnets were examined to see if the leads were properly tied down (to prevent flexing and strand breakage), if the G10 lead-clamping blocks were intact and were sufficiently far away from the end of the single-phase can, and whether the beam tube insulation was tied down. All the magnets in six houses ( $A 1, A 2, A 3, A 4, B 1$, and $E 1)$, out of 24 houses in the ring, were examined, with 138 out of 200 magnets undergoing some degree of repair. Seven magnets were found to have broken lead strands and so were replaced.

\section{DO Dverpass Modifications}

A second major undertaking during this three month shutdown was the installation of a re-designed Main Ring overpass in the Do interaction region.a The original overpass was installed in 1984 and was intended to be more of a "proof-of-concept" test bed than a permanent operational feature. A known shortcoming of the original design was an undesirable increase in the vertical dispersion function around the Main Ring and a dispersion mis-match between the Main Ring and the Tevatron (leading to enittance growth in the Tevatron). The new design lengthened the overpass by some 700 feet (although the height remained the same) and incorporated a near-clone of the Bo "double dogleg" vertical bending system.

\section{Collider Operation}

The report on Collider Operation at the 1987 Conferencea came in the middle of the first actual physics run; the average store luminosity, duration, and quality are much higher now than they were then, and so are our expectations for the machine.

\section{Collider Run}

The 1987 run ended on May 11, having provided some 70 inverse nanobarns of integrated luminosity to CDF in $\sim 900$ hours of storage time. The peak initial luminosity attained was $\sim 1.5 \times 10^{29} \mathrm{~cm}^{-2} \mathrm{sec}^{-1}$.

\section{Collider Run}

Anti-protons were injected into the Tevatron on June 6 , and by June 12 , the first $1.8 \mathrm{TeV} 6 \times 6$ store was circulating with an initial luminosity of $4 \times 10^{28}$. $(6 \times 6$ is shorthand for 6 proton bunches colliding with 6 anti-proton bunches; all of the 1987 run was $3 \times 3$.) Dperation was sporadic for the first four weeks, but by week 6 integrated luminosity had surpassed the entire 1987 run. Since then, the performance of the Tevatron has been nothing short of phenomenal - a luminosity of $3 \times 1029$ (the "operational goa ") was reached on July 28 and the design luminosity of $1 \times 1030$ was achieved on September 7 . An integrated luminosity of $1000 \mathrm{nb}^{-1}$ (1 inverse picobarn) was delivered by September 24, $2000 \mathrm{nb} 1$ by November 5 , and $3000 \mathrm{nb}^{-1}$ by December 4. At present, the integrated luminosity is greater than $6 \mathrm{pb}^{-1}$, the peak luminosity has exceeded $2 \times 1030$, and store duration is averaging greater than 14 hours. Figure 2 shows the integrated luminosity through March 12, 1989, while figure 3 shows the progression of the peak initial luminosity.

\section{Tevatron Collider Operation}

Integrated Luminosity at $900 \mathrm{GeV}$

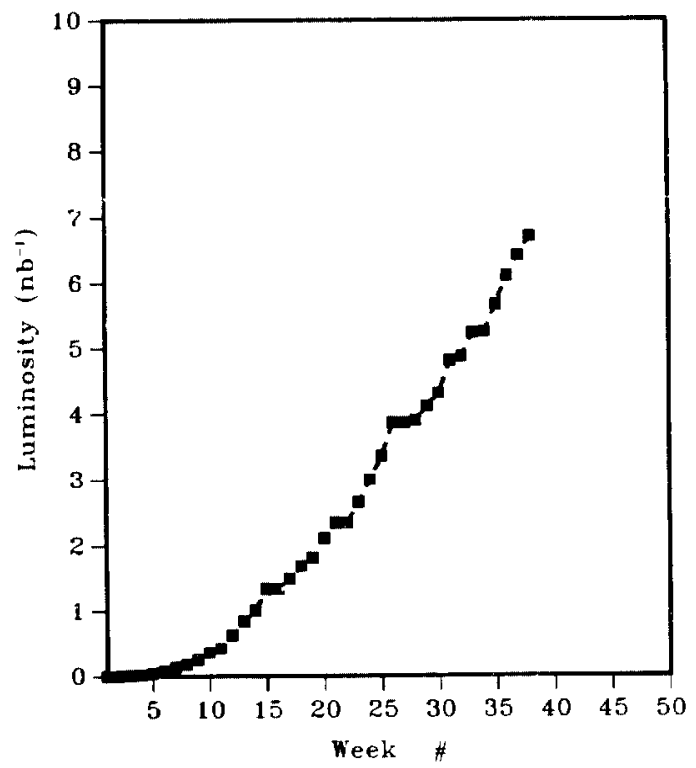

Figure 2: Integrated luminosity from $6 / 20 / 88-3 / 12 / 89$

Meanwhile, the $\mathrm{P}$-bar Source has not been resting on its laurels - the peak stack achieved exceeds $86 \times 1010$ anti-protons, the number of p-bars stacked in one week has reached $175.5 \times 1010$, and the hourly stacking rate has been as high as $2.1 \times 1010$. The Accumulator has maintained the anti-proton stack for as long as 39 days.

The present run is delivering integrated luminosity at a rate which is about twenty times greater than that delivered during the first collider run; this spectacular difference is not (entirely) the result of luck.4 There are more anti-protons available this run. Main Ring and Accumulator improvements have resulted in a very much improved stacking rate. For instance, Main Ring intensity has averaged about $1.7 \times 10^{12} \mathrm{ppp}$ on stacking cycles, compared to $1.2 \mathrm{x}$ 1012 last run; in the $\mathrm{P}$-bar source, the Accumulator aperture was increased to the design 
value and the horizontal dispersion in the low dispersion straights was corrected, core cooling was improved due to the intraduction of microwave mode dampers, and Debuncher betatron cooling times were reduced by the addition of optical notch filters. These improvements, coupled with improved Tevatron reliability, have resulted in larger stacks. The larger stacks have made it profitable to extract bunches six times instead of only three times from the Accumulator. Once the bunches have been extracted, the improved Main Ring transmission and coalescing have resulted in higher intensity single bunches at $150 \mathrm{GeV}$. These improvements have resulted in an overall gain factor of about seven.

\section{Tevatron Collider Operation}

Peak Luminosity/Day

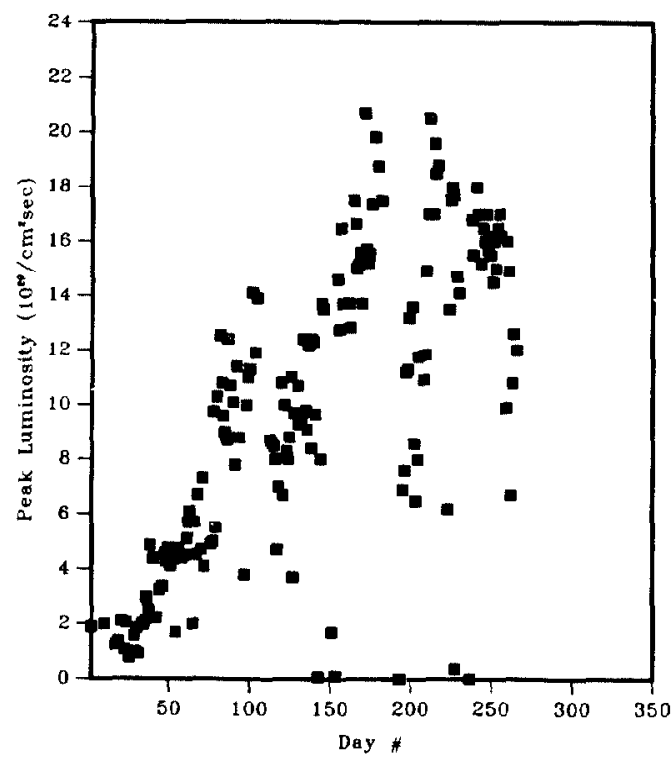

Figure 3: Peak luminosity per day

Getting particles into a single high intensity bunch in the Main Ring at $150 \mathrm{GeV}$ is only part of what is required for large integrated luminosity. In the first collider run, one of the major problems was not being able to obtain sufficiently small beam sizes in the Tevatron and, even if they had been small enough, not being able to keep them small during a store. The transverse emittances of the beans in the Tevatron during the present run are half as large as in the last one. This is because the vertical dispersion match between the Main Ring and Tevatron was improved by the re-configuration of the DO overpass, and because the compensation of the time-dependent higher order multipole fields in the Tevatron magnets has been greatly improved. The abort kicker power supplies were identified as the primary source of the anomalous transverse emittance growth during the last run; modifications to these supplies have resulted in an improvement in the luminosity lifetime by a factor of 2 to 3 . Unce these kickers were fixed, the full advantage of the "100\% mini-beta" squeeze could be implemented and this reduced the beam size at CDF even further. Without these improvements, the present run's integrated luminosity nould be smaller by another factor of three

\section{Reliability}

Obviously, much of the present run's success is duc to the outstanding reliability of the accelerator since the start up in June; during the past 25 weeks, the accelerator has been engaged in beam storage or set up for $\bar{p}-p$ transfers for an average of $69.5 \%$ of the scheduled time each week. The remaining $30 \%$ is an amalgam of equipment failures, scheduled and ad hoc studies, and store loss investigation. Of the roughly 2000 hours of equipment downtime (out of $\sim 6400$ hours available thus far), Tevatron systems have accounted for 44\% of the total, Main Ring and Anti-Proton Source combined for $35 \%$, Linac and Booster for $6 \%$, Controls for $51 / 2 \%$ and Utilities for $31 / 2 \%$; about $1 / 3$ of the Teratron downtime is due to the two dipole failures which have occurred this run (these were both leaks in the insulating vacuum and do not seem to be related to the failures experienced during the fixed target run).

\section{Acknowledgements}

There have been many operational improvements which are necessarily invisible if they are successful. These invisible improvements are nonetheless very important to the integrated luminosity. One of these items is the policy of relentless pursuit of things which kill stores. The improved diagnostics, controls, and applications software needed to accomplish this for each killed store involves the continuing efforts of many individuals. Without question, it is their constant effort which keeps the collider functioning as well as it does.

\section{The Future}

The present collider run is slated to end on June 1 with a hoped-for goal of $10 \mathrm{pb}^{-1}$ delivered Iuminosity; following a few weeks of studies, the accelerator will enter a five month $M \& D$ period to complete the repair program on the remaining Tevatron dipoles. Start up for another $800 \mathrm{GeV}$ fixed-target run should begin around December 1.

\section{References}

1. B. Hanna, H. Jostlein, D. Plant, S. Pruss, "InSitu Inspection of Superconducting Dipoles in the Tevatron", H2O, this conference.

2. D. Trbojevic, R. Gerig, "Design and Commissioning of the DO Non-Dispersive Overpass in the Fermilab Main Ring", X15, this conference.

3. R. Johnson, "Initial Operation of the Tevatron Collider", invited paper, 1987 Particle Accelerator Conference, Vancouver, B.C.

4. D. Finley, private communication. 